\title{
Effect of inversion-layer height and Coriolis forces on developing wind-farm boundary layers
}

\author{
D. Allaerts* and J. Meyers ${ }^{\dagger}$ \\ Department of Mechanical Engineering, KU Leuven, \\ Celestijnenlaan 300-bus 2421, B3001 Leuven, Belgium
}

\begin{abstract}
Offshore boundary layers are often neutrally stratified and capped by an inversion layer. In the current study, large-eddy simulations are used to investigate the effect of inversionlayer height and Coriolis forces on developing wind-farm boundary layers. The pseudospectral LES code SP-Wind combined with the concurrent-precursor method are used to model the wind farm. We find that internal boundary layer growth follows the typical 4/5 law as long as the inversion is located high above the wind farm. Further, a change in flow direction occurs throughout the wind farm, which is caused by the decrease in Coriolis forces due to the deceleration of the flow in the farm. This effect becomes more pronounced in low boundary layers, and can result in considerable turbine wake deflection near the end of the farm. We also observe differences of up to $17 \%$ in power deficit in downstream turbines when decreasing the inversion-layer height from 1000 to $250 \mathrm{~m}$. The latter is a height often found in offshore boundary layers.
\end{abstract}

\section{Nomenclature}

$C_{T}^{\prime} \quad$ Disk-based thrust coefficient

$f_{c} \quad$ Coriolis parameter

$f_{i} \quad$ Body force representing the effect of wind turbines

$h \quad$ Inversion base height

$\Delta h \quad$ Inversion thickness

$q_{j}^{s g s}$ Subgrid-scale heat flux

$s_{x}, s_{y}$ Streamwise and spanwise turbine spacing

$\tilde{u}_{i} \quad$ Filtered velocity component $(\mathrm{i}=1,2,3)$

$u_{*} \quad$ Friction velocity

$x_{i} \quad$ Coordinate direction $(\mathrm{i}=1,2,3)$

$z_{h} \quad$ Turbine hub height

Greek

$\tilde{\theta} \quad$ Filtered potential temperature

$\Delta \theta \quad$ Capping inversion strength (temperature jump)

$\tau_{i j}^{r} \quad$ Subgrid-scale stress tensor

\section{Introduction}

Modern wind energy extraction by means of horizontal axis wind turbines tends towards centralized production in large onshore and offshore wind farms, and the amount of turbines inside wind farms is likely to grow even more in the future. In large wind farms extending over several kilometres, the interaction with the atmospheric boundary layer (ABL) can no longer be neglected. Over the last decade, multiple studies have have been dedicated to the interaction between wind farms and the $\mathrm{ABL},{ }^{1-5}$ taking into account various

\footnotetext{
*Electronic mail: dries.allaerts@kuleuven.be

${ }^{\dagger}$ Electronic mail: johan.meyers@kuleuven.be
} 
aspects of atmospheric turbulence. In the current study, large-eddy simulations (LES) of large wind farms in the $\mathrm{ABL}$ are performed, taking into account stratification and rotation effects.

The atmospheric boundary layer considered here will be of the conventionally neutral type. This term was introduced to indicate the neutral boundary layer developing against a stably stratified background state, and contrasts with the truly neutral ABL. ${ }^{6}$ Over land, conventionally neutral boundary layers (CNBL) are only found during a short transition period after sunset or in overcast conditions with very strong winds..$^{7,8}$ By contrast, offshore CNBLs occur more often as the surface heat flux tends to be smaller at sea. ${ }^{9}$ Under certain conditions, CNBLs can also form above large lakes or semi-enclosed seas (e.g., the Baltic Sea) due to boundary-layer air advection from land. Especially during daytime, when the land is heated by the sun, and in early spring, when the water temperature is still relatively low, the air will warm up over land and flow out over the colder water. The change in surface roughness and heat flux will result in the growth of a stable internal boundary layer, which eventually develops into a neutral boundary layer capped by an inversion layer. This flow regime has been observed and analysed in several studies. ${ }^{10-12}$

Figure 1 shows a schematic representation of the conventionally neutral atmospheric boundary layer. The
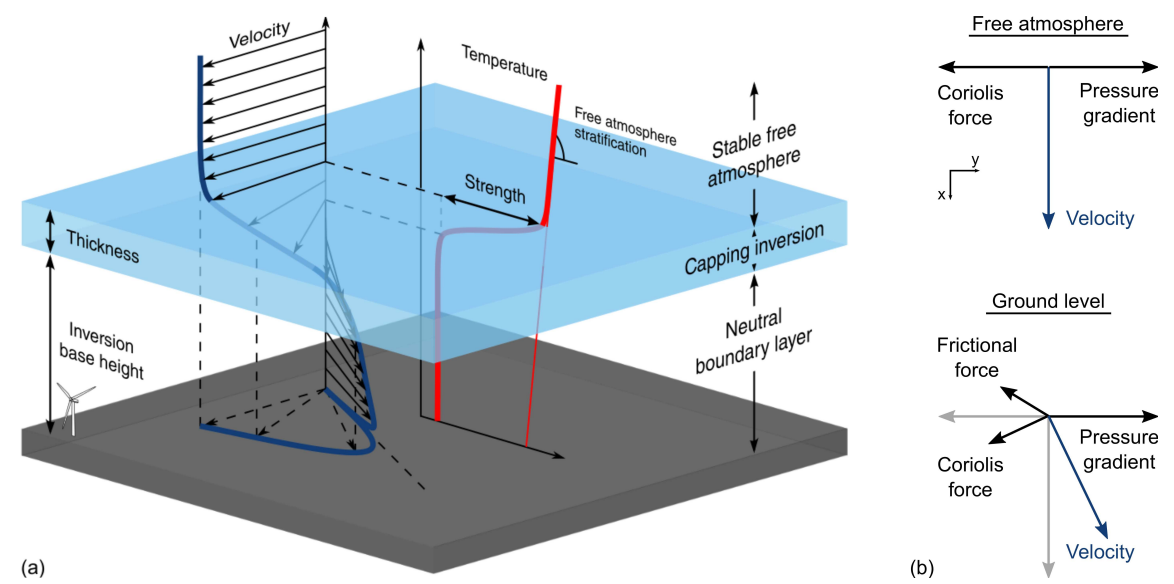

Figure 1. Schematic representation of the conventionally neutral atmospheric boundary layer, showing (a) profiles of the potential temperature and the velocity vector as a function of height from a three-dimensional point of view and (b) plane view of the horizontal force balance in the free atmosphere and at ground level.

upper part of the CNBL is the stably stratified free atmosphere, where the flow is non-turbulent and the potential-temperature gradient is constant. In this region, the wind speed results from the balance between the horizontal pressure gradient and the Coriolis force, as shown in figure 1(b). Near the surface lies the neutral, turbulent boundary layer, characterized by a constant potential-temperature profile and zero surface heat flux. Here, the wind speed decreases towards the ground, and, as a result, rotates towards the pressure gradient due to the decreasing Coriolis force (see figure 1(b)). At the interface between the neutral boundary layer and the free atmosphere, a thin inversion layer or capping inversion can often be found. Inside this layer, large discontinuities in potential temperature and velocity appear, giving the layer a strong thermal stability. Consequently, entrainment at the top of the boundary layer by upward moving turbulent drafts is reduced considerably, making the inversion layer act like a rigid lid on top of the boundary layer.

In a prior study, we showed that the capping inversion has an important impact on wind-farm performance for the fully developed case ${ }^{13}$ as it effectively controls the height and the growth of the boundary layer. In this study, the effect of inversion-layer height and Coriolis forces on developing wind-farm boundary layers will be investigated by varying the inversion-layer properties of the undisturbed flow.

The remainder of this study is organized as follows. In section II, a brief overview of the LES methodology is presented and the setup of the various cases is discussed. Section III describes the main trends in developing wind-farm boundary layer, and conclusions are drawn in section IV. 


\section{Methodology and Case Setup}

\section{A. Governing equations and LES code}

In LES of stratified, rotational atmospheric turbulence, the governing equations are the filtered transport equations for mass, momentum and potential temperature:

$$
\begin{gathered}
\frac{\partial \tilde{u}_{i}}{\partial x_{i}}=0 \\
\frac{\partial \tilde{u}_{i}}{\partial t}+\tilde{u}_{j} \frac{\partial \tilde{u}_{i}}{\partial x_{j}}=-\frac{1}{\rho_{0}} \frac{\partial \tilde{p}^{\star}}{\partial x_{i}}+\delta_{i 3} g \frac{\tilde{\theta}-\theta_{0}}{\theta_{0}}+f_{c} \epsilon_{i j 3} \tilde{u}_{j}-\frac{\partial \tau_{i j}^{r}}{\partial x_{j}}+f_{i}-\frac{1}{\rho_{0}} \frac{\partial p_{\infty}}{\partial x_{i}}, \\
\frac{\partial \tilde{\theta}}{\partial t}+\tilde{u}_{j} \frac{\partial \tilde{\theta}}{\partial x_{j}}=-\frac{\partial q_{j}^{s g s}}{\partial x_{j}},
\end{gathered}
$$

with $\tilde{u}_{i}$ and $\tilde{\theta}$ the filtered velocity and potential-temperature fields, respectively. Further, $\rho_{0}$ and $\theta_{0}$ correspond to the adiabatic background state and $f_{c}$ is the Coriolis parameter, which is set equal to $10^{-4} \mathrm{~s}^{-1}$. The modified pressure $\tilde{p}^{\star}=\tilde{p}-p_{\infty}+\rho_{0} \tau_{k k} / 3$ accounts for the trace of the subgrid-scale stress tensor, and $p_{\infty}$ is the applied background pressure. The residual subgrid-scale stress tensor $\tau_{i j}^{r}$ and the subgrid-scale heat flux $q_{j}^{\text {sgs }}$ are modelled with a stability dependent Smagorinsky model, ${ }^{14}$ and the wall stress is modelled using classic Monin-Obukhov similarity theory, i.e., ${ }^{15}$

$$
\begin{aligned}
& \tau_{w 1}=-\left(\frac{\kappa}{\ln z / z_{0}}\right)^{2}\left(\hat{\tilde{u}}^{2}+\hat{\tilde{v}}^{2}\right)^{0.5} \hat{\tilde{u}}, \\
& \tau_{w 2}=-\left(\frac{\kappa}{\ln z / z_{0}}\right)^{2}\left(\hat{\tilde{u}}^{2}+\hat{\tilde{v}}^{2}\right)^{0.5} \hat{\tilde{v}} .
\end{aligned}
$$

Finally, the wind turbines are modelled with a non-rotating actuator disk method (ADM).$^{2,16}$ In this method, the effect of the turbines on the flow is represented by the body forces $f_{i}$, where we set $f_{2}=f_{3}=0$ and

$$
f_{1}=-\frac{1}{2} C_{T}^{\prime}\left\langle\bar{u}^{T}\right\rangle_{d}^{2} \mathscr{R}(\boldsymbol{x}),
$$

with $C_{T}^{\prime}$ the disk-based thrust coefficient, $\left\langle\bar{u}^{T}\right\rangle_{d}$ the local disk-averaged and time-filtered velocity and $\mathscr{R}(\boldsymbol{x})$ a geometrical smoothing function. The full details of the pseudo-spectral LES code SP-Wind, used to solve these equations numerically, can be found in Refs. 2 and 13.

In the horizontal direction, the spectral scheme implies periodic boundary conditions. Therefore, standard simulations of wind turbines represent the asymptotic limit of an "infinite" farm. Entrance effects and streamwise evolution in wind farms of finite length can be simulated by circumventing the domain's periodicity, which is achieved by applying the concurrent-precursor method ${ }^{17}$ (see figure 2). In this method, a fringe region is added to the domain, in which the flow is forced to match a desired inflow. In this way, the global periodicity is preserved while the region of interest is non-periodic. A fully-developed turbulent inflow profile with consistent coherent structures is obtained from an auxiliary simulation (i.e. the precursor), which runs simultaneously with the main simulation.

\section{B. Case Setup}

A suite of LES simulations is performed to investigate the effect of inversion-layer height and Coriolis forces on wind farms. The simulations are performed in three stages. In a first stage, a conventionally neutral atmospheric boundary layer without wind turbines is simulated in a normal periodic domain (the precursor domain in figure 2). The boundary layer is allowed to develop for 20 physical hours to reach a fully developed, statistically stationary regime. In a second stage, the concurrent-precursor method is applied. The precursor simulation starts from the velocity and temperature fields developed in the first stage, and produces inflow fields for the main domain containing the actual wind farm. The simulation is advanced for another two hours, corresponding to about three domain flow-through times, to allow any transitional effects from windfarm start-up to die out. In the last stage, statistics are collected over two hours. 


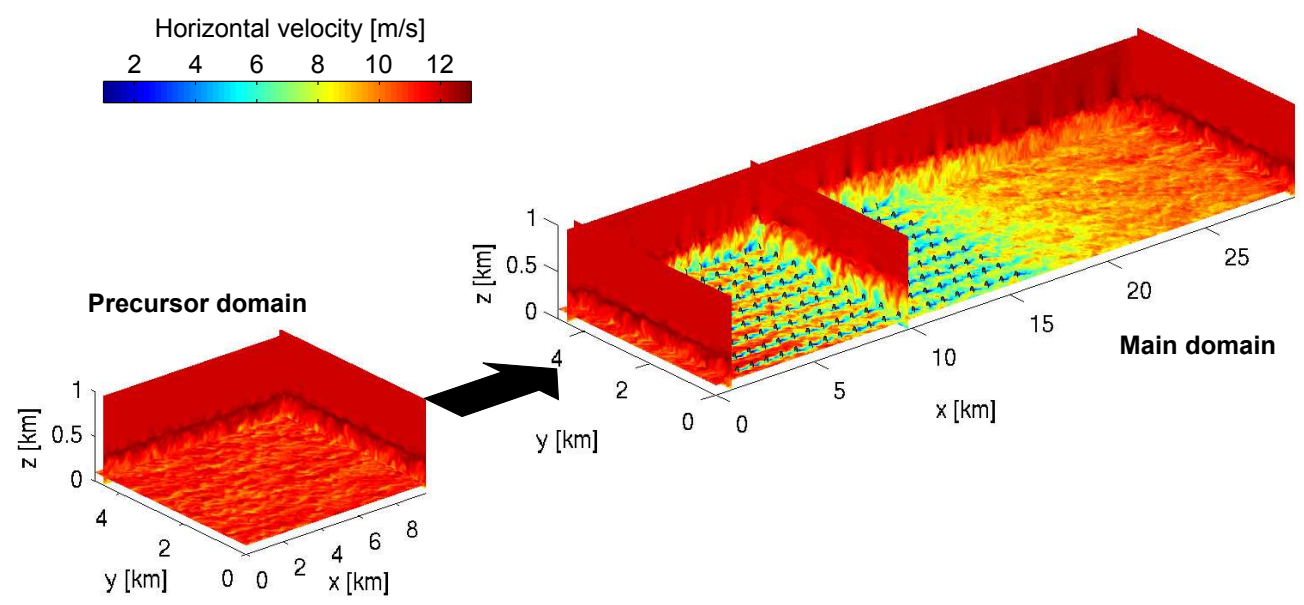

Figure 2. Schematic representation of the concurrent-precursor method.

Table 1. Numerical setup of LES simulations

\begin{tabular}{ccccccc} 
& \multicolumn{2}{c}{ Inversion layer } & \multicolumn{2}{c}{ Main domain } & \multicolumn{2}{c}{ Precursor domain } \\
& $h[\mathrm{~m}]$ & $\Delta \theta[\mathrm{K}]$ & $L_{x} \times L_{y} \times L_{z}[\mathrm{~km}]$ & $N_{x} \times N_{y} \times N_{z}$ & $L_{x} \times L_{y} \times L_{z}[\mathrm{~km}]$ & $N_{x} \times N_{y} \times N_{z}$ \\
\hline S10 & 1000 & 1.0 & $28.8 \times 4.8 \times 5.0$ & $960 \times 320 \times 400$ & $9.6 \times 4.8 \times 5.0$ & $320 \times 320 \times 400$ \\
S20 & 500 & 2.0 & $28.8 \times 4.8 \times 5.0$ & $960 \times 320 \times 320$ & $9.6 \times 4.8 \times 5.0$ & $320 \times 320 \times 320$ \\
S40 & 250 & 4.0 & $28.8 \times 4.8 \times 5.0$ & $960 \times 320 \times 320$ & $9.6 \times 4.8 \times 5.0$ & $320 \times 320 \times 320$
\end{tabular}

Details on the numerical setup of the various LES cases are provided in table 1 . The properties of the inversion layer are chosen such that the CNBL in the precursor is in equilibrium. To this end, the empirical formulation of Csanady ${ }^{18}$ for the asymptotic depth $h$ is used:

$$
h=A \frac{\theta_{0}}{g \Delta \theta} u_{*}^{2},
$$

with $A \approx 500$ an empirical parameter. ${ }^{10,18}$ Using typical values of $0.26-0.28 \mathrm{~m} / \mathrm{s}$ for the friction velocity over sea, we obtain the combinations of inversion height $h$ and inversion strength $\Delta \theta$ mentioned in table 1 . These cases cover the range of boundary-layer heights typically found in offshore boundary layers. ${ }^{13}$ Other input parameter are set to representative offshore values, i.e., a constant geostrophic wind speed $G=12 \mathrm{~m} / \mathrm{s}$ and a surface roughness length ${ }^{19} z_{0}=2 \times 10^{-4}$ are prescribed. The free atmosphere lapse rate $\gamma$ equals $1 \mathrm{~K} / \mathrm{km}$ and the temperature of the mixed layer is $\theta_{m}=15^{\circ}$, which is also taken as the reference temperature $\theta_{0}$. The initial potential-temperature profile is generated using a smooth curve proposed Rampanelli and Zardi ${ }^{20}$ in which the inversion thickness is set to $\Delta h=100 \mathrm{~m}$.

The wind farm under consideration consists of 180 turbines with rotor diameter $D=100 \mathrm{~m}$ and hub height $z_{h}=100 \mathrm{~m}$, arranged in an aligned pattern of 20 rows (streamwise) and 9 columns (spanwise). The disk-based thrust coefficient $C_{T}^{\prime}$ is set to $^{2} 4 / 3$, and the streamwise and spanwise spacing are $s_{x}=7.5 D$ and $s_{y}=5.33 D$, respectively. Hence, the streamwise extent of the wind farm is $15 \mathrm{~km}$. Initial results revealed that the domain should contain a sufficiently large region downstream of the farm for boundarylayer recovery, so the domain size is set to almost twice the size of the wind farm. Further, the height of the domain is set to $5 \mathrm{~km}$ to minimize blockage effects due to the upper boundary. The vertical grid resolution is $5 \mathrm{~m}$ in the lowest $1000 \mathrm{~m}$ of the domain (1500 $\mathrm{m}$ for case S10), and is stretched up to $40 \mathrm{~m}$ in the remainder of the domain. The precursor domain differs only in the streamwise dimension, which is one third of the main domain to reduce computational costs. In total, the simulations use approximately 130 million grid cells (164 million for case S10), for the combination of main and precursor domain. 

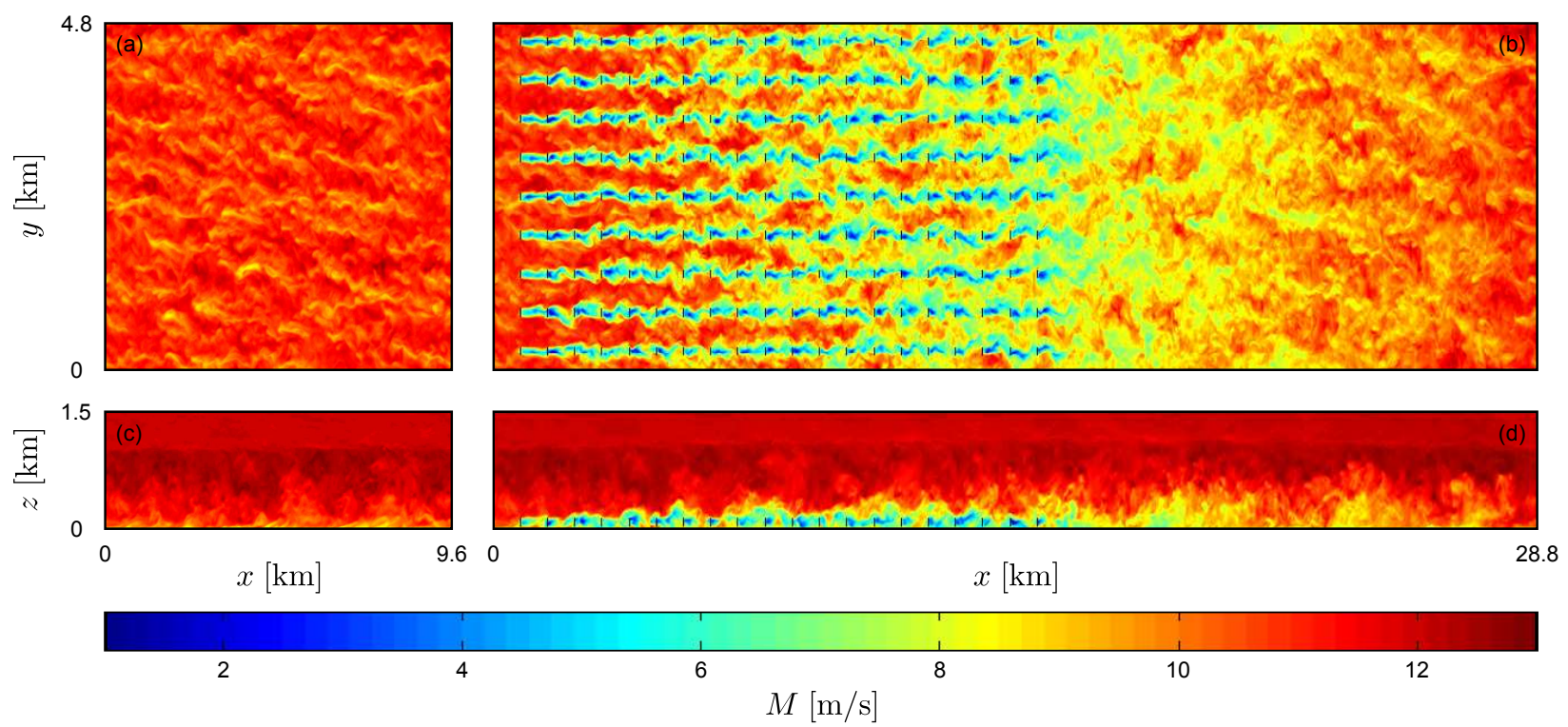

Figure 3. Instantaneous contours of horizontal velocity $M=\left(\tilde{u}^{2}+\tilde{v}^{2}\right)^{0.5}$, obtained at the end of the second initialization stage, for case S10; $(\mathbf{a}, \mathbf{b})$ An $\mathrm{x}-\mathrm{y}$ plane at turbine hub height $z_{h}=100 \mathrm{~m}$; (c,d) An x-z plane through the middle of a turbine column. The left pane shows the precursor domain (a,c) and the right pane shows the main domain $(b, d)$, where turbine-disk locations are indicated with vertical black lines.

\section{Developing wind-farm boundary layers}

A snapshot of the horizontal velocity through a developing wind farm is shown in figure 3 . The velocity contours correspond to a single instant in time at the end of the second initialization stage of case S10. On the left, figures $3(\mathrm{a})$ and $3(\mathrm{c})$ show an $\mathrm{x}-\mathrm{y}$ plane $\left(\right.$ at $z_{h}=100 \mathrm{~m}$ ) and an $\mathrm{x}-\mathrm{z}$ plane of the precursor domain. In the top view, typical elongated structures in streamwise direction are observed. The side view, on the other hand, shows that the turbulent ejection and sweep motions are confined by the inversion, which is located here at about $1 \mathrm{~km}$. The flow above the capping inversion is non-turbulent and shear free. Similar cross sections of the main domain are shown in figures 3(b) and 3(d), where turbine-disk locations are indicated with vertical black lines. Here we see the meandering of the turbine wakes and the gradual increase in velocity deficit throughout the farm. After about five turbine rows, spanwise wake interactions between different turbine columns start to occur. In the side view, the vertical extent of the turbine wakes increases downstream, and the height of the capping inversion appears to increase as well.

Figure 4 shows contours of time-averaged horizontal velocity in an x-z plane through the middle of a turbine column for the various LES simulations. In all cases, momentum deficit builds up through the farm and an internal boundary layer (IBL) develops. The height of this layer is calculated as the height where the ratio of the time-averaged velocity and the mean inflow velocity at the same height reaches a threshold, ${ }^{21}$ which we set here to $95 \%$. The IBL is shown as a dashed line in figure 4, together with the base and top of the inversion layer (solid lines). Although the velocity fields qualitatively look very similar, the shape of the internal boundary layer indicates that there are important differences in the boundary-layer flow between the three cases. For case S10, with inflow boundary-layer height at $1000 \mathrm{~m}$, the inversion layer is located far above the farm and does not interact with the internal boundary layer. However, when lowering the inflow inversion layer, the IBL collides with the inversion at some point and is limited in growth further downstream. In cases S20 and S40, we see that this event occurs at approximately the tenth and fifth turbine row, respectively.

The IBL height of the different cases is compared in figure 5(a) in a double logarithmic scale. In a truly neutral atmospheric boundary layer, the IBL growth typically follows a $4 / 5$ power law. ${ }^{22}$ Under conventionally neutral conditions, this theoretical prediction appears to hold well when the inversion is located high above the farm, i.e., in case S10 (from the fourth turbine row onwards). In cases S20 and S40, on the other hand, we clearly see a deviation from the theoretical profile when the IBL reaches the inversion layer. 

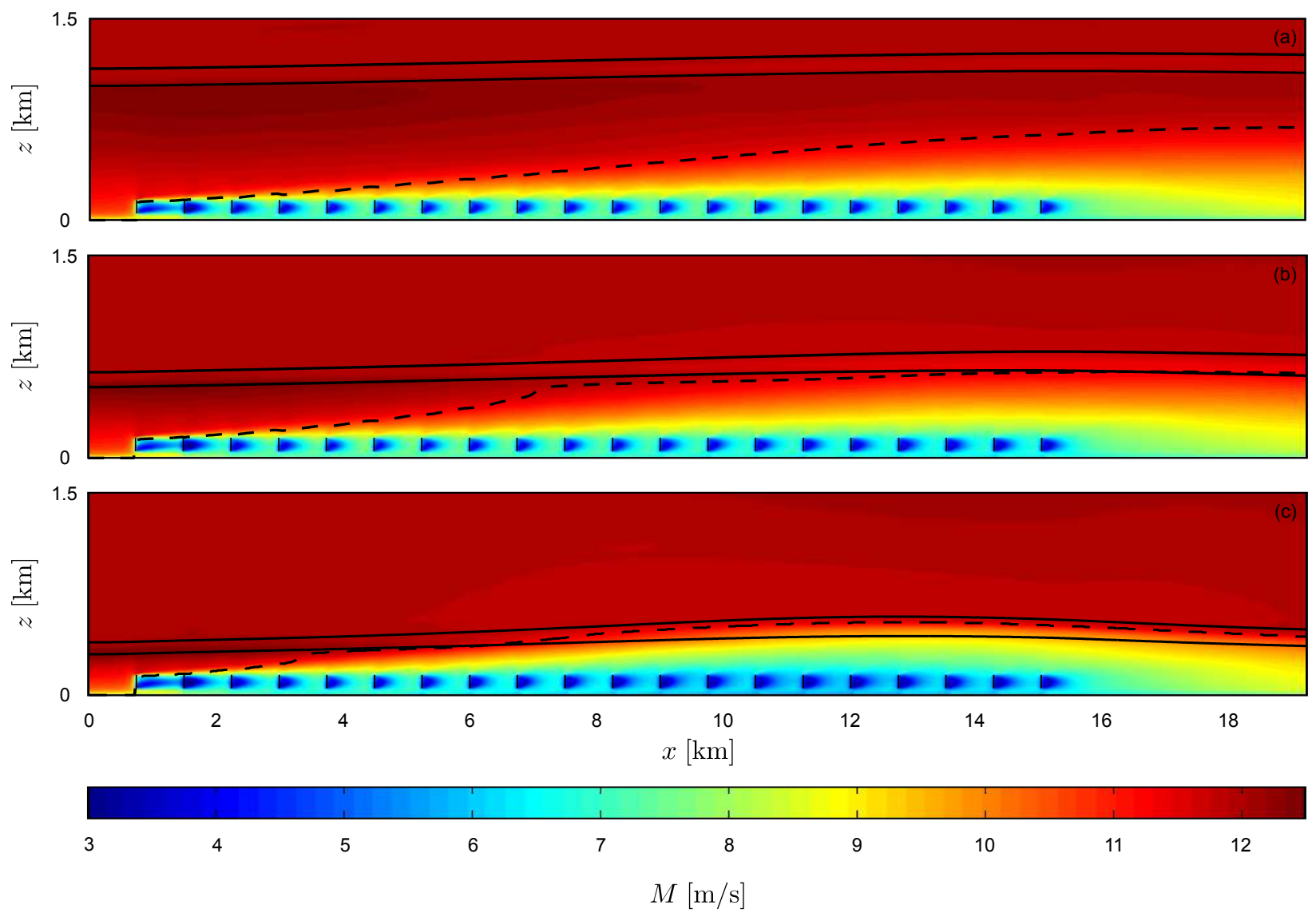

Figure 4. Time-averaged contours of horizontal velocity $M=\left(\tilde{u}^{2}+\tilde{v}^{2}\right)^{0.5}$ in an x-z plane through the middle of a turbine column, for cases S10 (a), S20 (b) and S40 (c). The evolution of the inversion layer base and top (solid lines) as well as the height of the internal boundary layer (dashed line) are included. The location of the wind-turbine disks are indicated with vertical black lines.

The momentum deficit in the wind farm is accompanied by a decrease in the streamwise mass flow rate. Due to the continuity equation, part of the mass flow is directed upwards and results in a thickening of the boundary layer, as can be seen in figure 4 . The displacement of the boundary-layer top $\eta$, defined as the difference between the actual boundary-layer height and the unperturbed height upstream of the farm, is shown in figure 5(b) for the various simulations. As the elevation of the upstream inversion layer is decreased, the boundary-layer displacement above the farm increases. For the wind farm under consideration, lowering the inversion from $1000 \mathrm{~m}$ to $250 \mathrm{~m}$ raises the maximum displacement from $113 \mathrm{~m}$ to $162 \mathrm{~m}$. Note that for the $250 \mathrm{~m}$ case, this corresponds to a displacement of $65 \%$.

The flow deceleration induces a second effect related to the Coriolis force and the horizontal force balance. As the Coriolis forces scale linearly with the wind velocity, the decrease in velocity magnitude causes the wind to turn towards the pressure gradient, similar to the vertical wind veer in a classical Ekman boundary layer. Figure 6(a) shows the difference in wind direction at hub height with respect to the inflow wind direction, averaged over the full spanwise direction and over a streamwise distance $s_{x} D$ centered around each turbine row. It shows that the flow direction changes almost linearly throughout the farm. At the end of the farm, the total wind veer is about $2-3^{\circ}$, where the highest wind veer corresponds to the lowest boundary-layer height. This result matches with the conclusions of previous studies on fully developed wind farms, for which it was shown that the geostrophic angle increases with decreasing boundary-layer height. ${ }^{13}$

The wind-turbine power extraction, averaged over turbine rows and normalized by the power of the first row, is shown in Figure 6(b). The power deficit in the farm appears to depend on the height of the incoming boundary layer, and increases for decreasing boundary-layer heights. The maximum (absolute) difference in power deficit between cases S10 and S40 is equal to $17.6 \%$. Surprisingly, a very small power increase is observed at the end of the farm in case S40 (3.4\% over the last four turbine rows), but not in case S10 or 

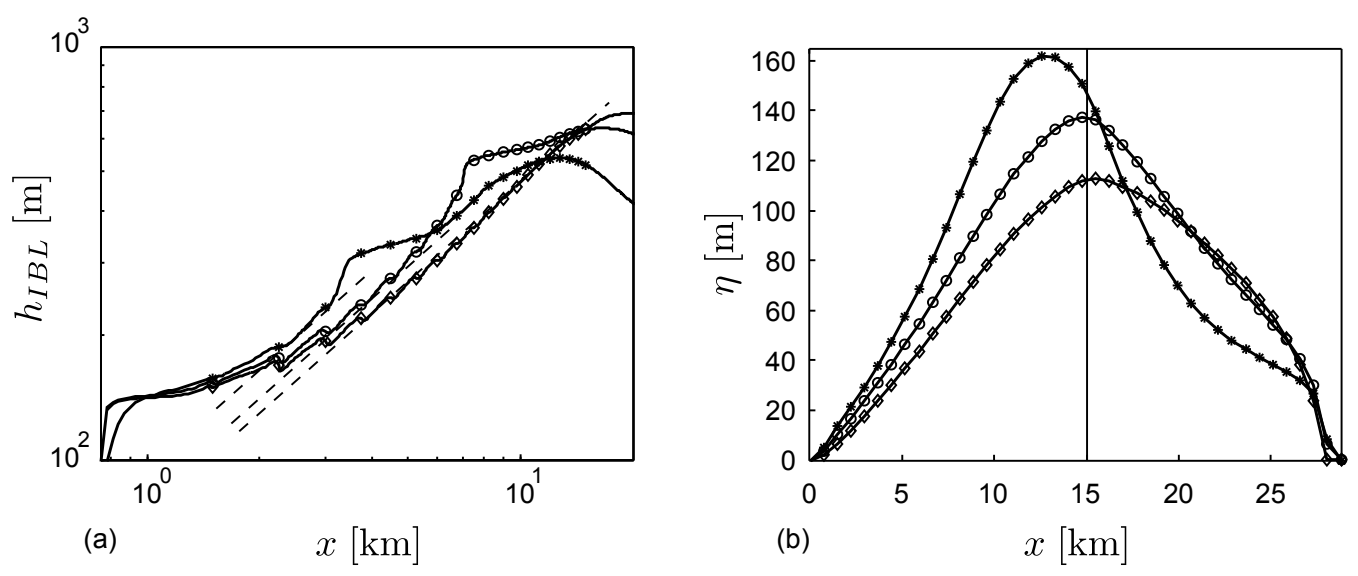

Figure 5. (a) Internal boundary layer height, shown in a double logarithmic scale, and (b) boundary-layer top displacement, for cases S10 (diamonds), S20 (circles) and S40 (stars). The dashed lines in (a) correspond to the theoretical growth of internal boundary layers, i.e. the $4 / 5$ power law, and the vertical solid line in (b) indicates the end of the wind farm.
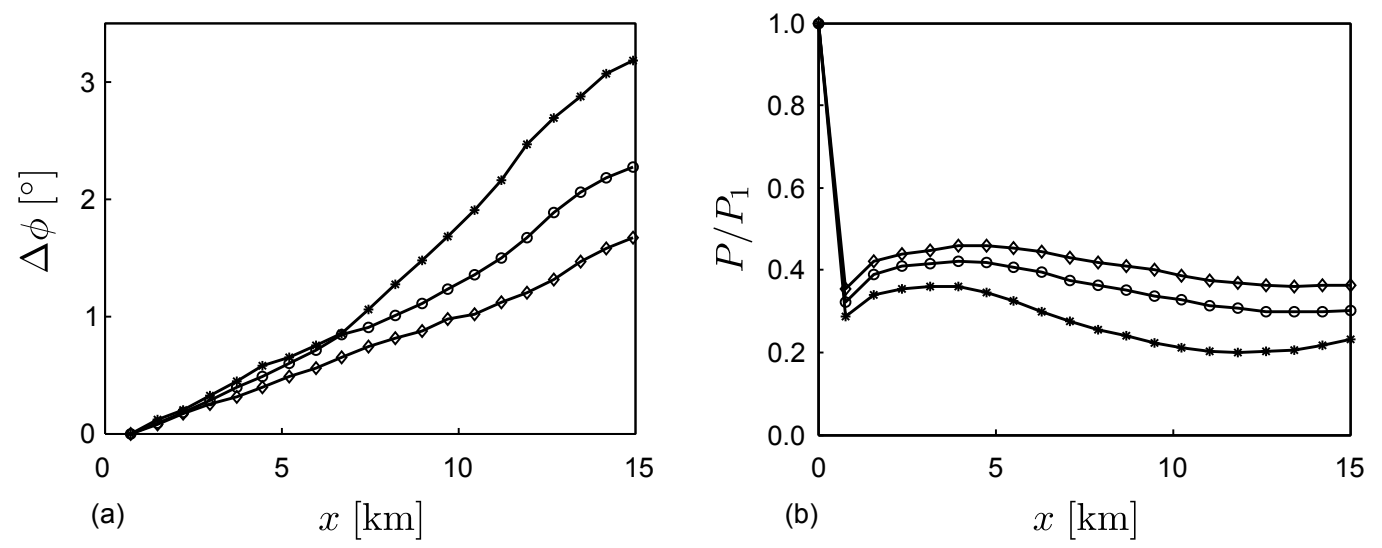

Figure 6. (a) Difference in wind direction at hub height with respect to the inflow wind direction, averaged over the full spanwise direction and over a streamwise distance $s_{x} D$ centered around each turbine row, and (b) average power extraction per turbine row, normalized by the first row, for cases S10 (diamonds), S20 (circles) and S40 (stars).

S20. This counter-intuitive behaviour is explained in figure 7, showing the time-averaged horizontal velocity contours in an x-y plane at hub height for case S40. In the detailed view of the last five turbines rows, we observe that the change in mean wind direction causes a relatively large deflection of the turbine wakes. Due to this deflection, the turbines are no longer located directly in the wake of the turbines in the row upstream. Instead, high speed winds from in between turbine columns are directed towards the rotor disks and cause an increase in power extraction.

\section{Conclusion}

The purpose of the present research was to investigate the effect of inversion-layer height and Coriolis forces on developing wind-farm boundary layers. A suite of LES simulations with varying inversion-layer heights is conducted using the LES code SP-Wind. To allow the study of streamwise developing features, the concurrent-precursor method is applied. The properties of the inversion layer upstream of the wind farm are set by choosing the inversion-layer height and strength in the precursor domain such that the CNBL is in equilibrium.

The results showed that the wind speed decreases gradually throughout the farm due to the energy extraction by the wind turbines. When the inversion layer is located high above the wind farm, the internal 


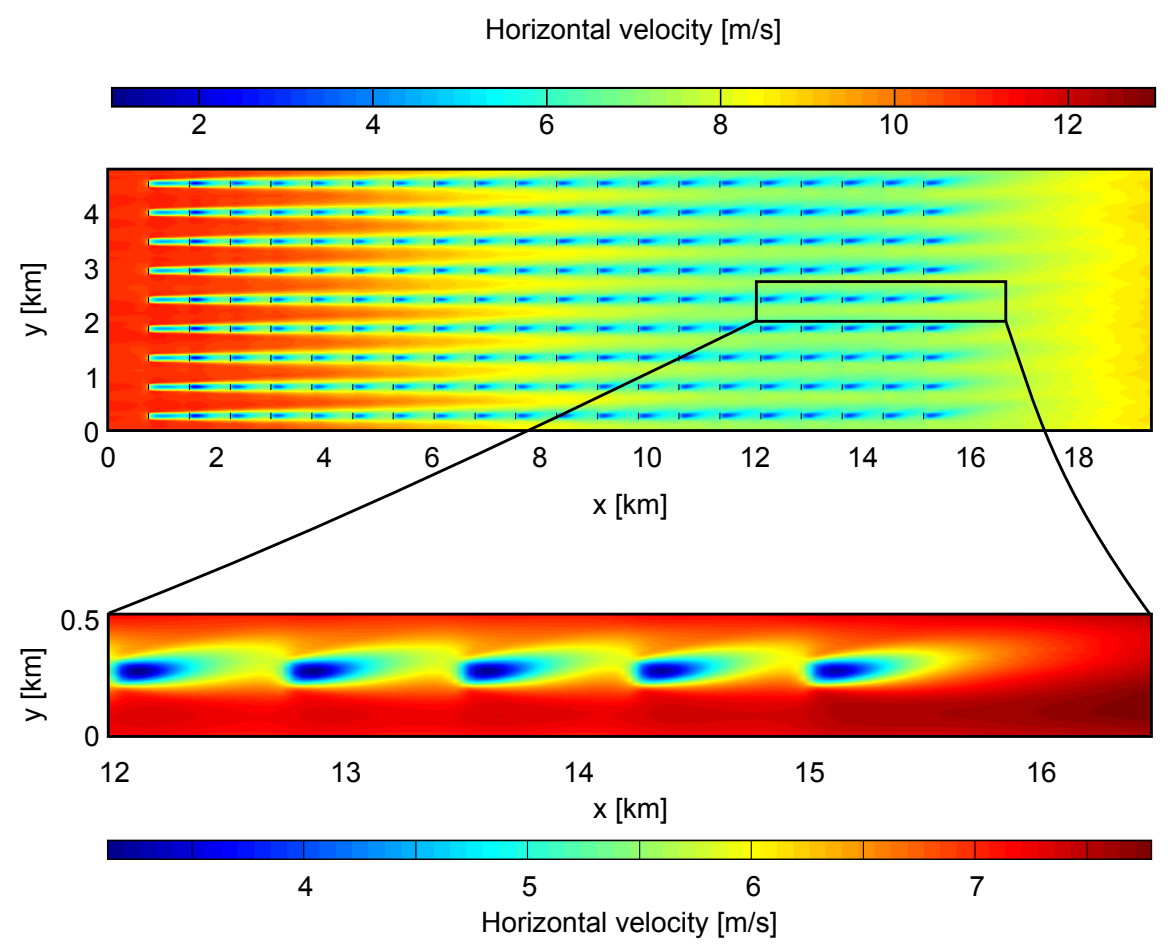

Figure 7. Full and detailed view of time-averaged horizontal velocity contours in an $x-y$ plane at hub height for case S40. The location of the wind-turbine disks are indicated with vertical black lines.

boundary layer developing above the farm follows the typical $4 / 5$ power law. However, significant deviation from this $4 / 5$ behaviour is observed when the height of the upstream boundary layer is decreased. It was also shown that the flow deceleration induces two additional effects. First, the top of the boundary layer is displaced due to the conservation of mass. Second, a change in flow direction occurs throughout the wind farm due to the Coriolis effect, with an increased total wind veer for decreasing inversion-layer heights. Finally, it was shown that the power deficit in downstream rows increases with decreasing inversion heights as well. Under certain conditions, an increase of power production can be observed near the end of the farm due to a strong wake deflection induced by Coriolis effects.

\section{Acknowledgments}

The authors acknowledge support from the European Research Council (FP7-Ideas, grant no. 306471). The computational resources and services used in this work were provided by the VSC (Flemish Supercomputer Center), funded by the Hercules Foundation and the Flemish Government - department EWI.

\section{References}

${ }^{1}$ Frandsen, S., Barthelmie, R., Pryor, S., Rathmann, O., Larsen, S., Højstrup, J., and Thøgersen, M., "Analytical modelling of wind speed deficit in large offshore wind farms," Wind Energy, Vol. 9, No. 1-2, 2006, pp. 39-53.

${ }^{2}$ Calaf, M., Meneveau, C., and Meyers, J., "Large eddy simulation study of fully developed wind-turbine array boundary layers," Phys. Fluids, Vol. 22, 2010, pp. 015110.

${ }^{3}$ Johnstone, R. and Coleman, G. N., "The turbulent Ekman boundary layer over an infinite wind-turbine array," J. Wind Eng. Ind. Aerodyn., Vol. 100, No. 1, 2012, pp. 46-57.

${ }^{4} \mathrm{Lu}, \mathrm{H}$. and Porté-Agel, F., "Large-eddy simulation of a very large wind farm in a stable atmospheric boundary layer," Phys. Fluids, Vol. 23, No. 6, 2011, pp. 065101.

${ }^{5}$ Abkar, M. and Porté-Agel, F., "The Effect of Free-Atmosphere Stratification on Boundary-Layer Flow and Power Output from Very Large Wind Farms," Energies, Vol. 6, No. 5, 2013, pp. 2338-2361.

${ }^{6}$ Zilitinkevich, S. S. and Calanca, P., "An extended similarity theory for the stably stratified atmospheric surface layer," Q. J. R. Meteorol. Soc., Vol. 126, No. 566, 2000, pp. 1913-1923.

${ }^{7}$ Stull, R. B., An Introduction to Boundary Layer Meteorology, Springer, 1988. 
${ }^{8}$ Garratt, J. R., The atmospheric boundary layer, Cambridge University Press, 1992.

${ }^{9}$ Businger, J. A. and Charnock, H., "Boundary Layer Structure in Relation to Larger-Scale Flow: Some Remarks on the JASIN Observations," Philos. Trans. R. Soc. London. Series A, Mathematical and Physical Sciences, Vol. 308, No. 1503, 1983, pp. 445-449.

${ }^{10}$ Tjernström, M. and Smedman, A.-S., "The vertical turbulence structure of the coastal marine atmospheric boundary layer," J. Geophys. R.: Oceans, Vol. 98, No. C3, 1993, pp. 4809-4826.

${ }^{11}$ Smedman, A.-S., Bergström, H., and Grisogono, B., "Evolution of stable internal boundary layers over a cold sea," J. Geophys. Res., Vol. 102, No. C1, 1997, pp. 1091-1099.

${ }^{12}$ Lange, B., Larsen, S., Højstrup, J., and Barthelmie, R., "The Influence of Thermal Effects on the Wind Speed Profile of the Coastal Marine Boundary Layer," Boundary-Layer Meteorol., Vol. 112, No. 3, 2004, pp. 587-617.

${ }^{13}$ Allaerts, D. and Meyers, J., "Large eddy simulation of a large wind-turbine array in a conventionally neutral atmospheric boundary layer," Phys. Fluids, Vol. 27, 2015, pp. 065108.

${ }^{14}$ Stevens, B., Moeng, C.-H., and Sullivan, P. P., "Entrainment and Subgrid Lengthscales in Large-Eddy Simulations of Atmospheric Boundary-Layer Flows," IUTAM Symposium on Developments in Geophysical Turbulence, edited by R. M. Kerr and Y. Kimura, Vol. 58 of Fluid Mechanics and Its Applications, Springer Netherlands, 2000, pp. 253-269.

${ }^{15}$ Moeng, C.-H., "A Large-Eddy-Simulation Model for the Study of Planetary Boundary-Layer Turbulence," J. Atmos. Sci., Vol. 41, No. 13, 1984, pp. 2052-2062.

${ }^{16}$ Meyers, J. and Meneveau, C., "Large eddy simulations of large wind-turbine arrays in the atmospheric boundary layer," AIAA Paper No. 2010-827, 2010.

${ }^{17}$ Stevens, R. J. A. M., Graham, J., and Meneveau, C., "A concurrent precursor inflow method for Large Eddy Simulations and applications to finite length wind farms," Renewable Energy, Vol. 68, 2014, pp. 46 - 50.

${ }^{18}$ Csanady, G. T., "Equilibrium theory of the planetary boundary layer with an inversion lid," Boundary-Layer Meteorol., Vol. 6, No. 1-2, 1974, pp. 63-79.

${ }^{19}$ Sullivan, P. P., Edson, J. B., Hristov, T., and McWilliams, J. C., "Large-Eddy Simulations and Observations of Atmospheric Marine Boundary Layers above Nonequilibrium Surface Waves," J. Atmos. Sci., Vol. 65, No. 4, 2008, pp. 1225-1245.

${ }^{20}$ Rampanelli, G. and Zardi, D., "A Method to Determine the Capping Inversion of the Convective Boundary Layer," J. Appl. Meteor., Vol. 43, No. 6, 2004, pp. 925-933.

${ }^{21} \mathrm{Wu}$, Y.-T. and Porté-Agel, F., "Simulation of Turbulent Flow Inside and Above Wind Farms: Model Validation and Layout Effects," Boundary-Layer Meteorol., Vol. 146, No. 2, 2013, pp. 181-205.

${ }^{22}$ Elliott, W. P., "The growth of the atmospheric internal boundary layer," Trans. Am. Geophys. Union, Vol. 39, No. 6, 1958, pp. 1048-1054. 